\title{
Article
}

\section{Tier-specific evolution of match performance characteristics in the English Premier League: It's getting tougher at the top}

Bradley, P.S., Archer, D., Hogg, B., Schuth, G., Bush, M., Carling, C., and Barnes, $\mathrm{C}$.

Available at http://clok.uclan.ac.uk/12457/

Bradley, P.S., Archer, D., Hogg, B., Schuth, G., Bush, M., Carling, C., ORCID: 0000-0002-7456-3493 and Barnes, C. (2016) Tier-specific evolution of match performance characteristics in the English Premier League: It's getting tougher at the top. Journal of Sports Sciences, 34 (10). pp. 980-987. ISSN 0264-0414

It is advisable to refer to the publisher's version if you intend to cite from the work. http://dx.doi.org/10.1080/02640414.2015.1082614

For more information about UCLan's research in this area go to http://www.uclan.ac.uk/researchgroups/ and search for < name of research Group>.

For information about Research generally at UCLan please go to http://www.uclan.ac.uk/research/

All outputs in CLoK are protected by Intellectual Property Rights law, including Copyright law. Copyright, IPR and Moral Rights for the works on this site are retained by the individual authors and/or other copyright owners. Terms and conditions for use of this material are defined in the policies page. 
This is a pre-proof corrected manuscript, as accepted for publication, of an article published by Taylor \& Francis in Journal of Sports Sciences on $11^{\text {th }}$ September 2015, available online:

http://dx.doi.org/10.1080/02640414.2015.1082614

PLEASE REFER TO THE PUBLISHED VERSION FOR CITING PURPOSES

Tier-Specific Evolution of Match Performance Characteristics in the English Premier League: It's Getting Tougher at the Top

Paul S. Bradley ${ }^{1}$, David Archer ${ }^{2}$, Bob Hogg ${ }^{2}$, Gabor Schuth ${ }^{2}$, Michael Bush ${ }^{2}$, Chris Carling ${ }^{3,4}$ and Chris Barnes ${ }^{5}$

${ }^{1}$ Carnegie School of Sport, Leeds Beckett University, UK;

${ }^{2}$ Department of Sport and Exercise Sciences, University of Sunderland, UK;

${ }^{3}$ LOSC Lille Métropole Football Club, Camphin-en-Pévéle, France;

${ }^{4}$ Institute of Coaching and Performance, University of Central Lancashire, UK

${ }^{5}$ Medical Department, West Bromwich Albion Football Club, UK

Running Title: Evolution and League Ranking. 


\section{Abstract}

This study investigated the evolution of physical and technical performances in the English Premier League (EPL) with special reference to league ranking. Match performance observations $(n=14700)$ were collected using a multiple-camera computerised tracking system across seven consecutive EPL seasons (2006-07 to 2012-13). Final league rankings were classified into Tiers: (A) $1^{\text {st }}-4^{\text {th }}$ ranking $(n=2519)$, (B) $5^{\text {th }}-8^{\text {th }}$ ranking $(n=2965),(C) 9^{\text {th }}-$ $14^{\text {th }}$ ranking $(n=4448)$ and (D) $15^{\text {th }}-20^{\text {th }}$ ranking $(n=4768)$. Teams in Tier B demonstrated moderate increases in high-intensity running distance while in ball possession from the 200607 to 2012-13 season $(P<0.001$; Effect Size [ES]: 0.68), with Tiers A, C and D producing less pronounced increases across the same period $(P<0.005$; ES: $0.26,0.41,0.33)$. Large increases in sprint distance were observed from the 2006-07 to 2012-13 season for Tier B $(P<0.001$; ES: 1.21$)$ while only moderate increases were evident for Tiers A, C and D $(P<0.001$; ES: $0.75,0.97,0.84)$. Tier B demonstrated large increases in the number of passes performed and received in 2012-13 compared to 2006-07 ( $P<0.001$; ES: $1.32-1.53)$ with small-to-moderate increases in Tier A $(P<0.001$; ES: 0.30-0.38), Tier C $(P<0.001$; ES: 0.46 $0.54)$ and Tier D $(P<0.001$; ES: $0.69-0.87)$. The point's difference between Tiers A and B in the 2006-07 season was 8 points but this decreased to just a single point in the 2012-13 season. The data demonstrate that physical and technical performances have evolved more in Tier B than any other Tier in the EPL and could indicate a narrowing of the performance gap between the top two Tiers.

Keywords: Longitudinal, football, standard, passing, sprinting. 


\section{Introduction}

Soccer is an intermittent sport with a myriad of physical and technical indicators influencing performance (Stølen, Chamari, Castagna, \& Wisløff, 2005). The physical demands of elite match play are well reported with factors such as physical capacity (Krustrup et al., 2003, 2005), context (Castellano, Blanco-Villasenor, \& Alvarez, 2011), technical level (Rampinini, Impellizzeri, Castagna, Coutts, \& Wisløff, 2009), team formation (Bradley et al., 2011), the standard of opponent (Rampinini, Coutts, Castagna, Sassi, \& Impellizzeri, 2007), seasonal period (Mohr, Krustrup, \& Bangsbo, 2003; Rampinini et al., 2007), fatigue/pacing (Bradley \& Noakes, 2013), surface (Andersson, Ekblom, \& Krustrup, 2008) and the environment (Mohr, Nybo, Grantham, \& Racinais, 2012) potentially impacting on match running performances. Despite this coverage, there is limited evidence supporting a link between success in soccer and match running performance or physical capacity (Apor, 1988; Mohr et al., 2003; Rampinini et al., 2007). The assertion that league ranking or competitive standard is related to a greater physical capacity or distance covered in matches is far too simplistic (Carling, 2013). For instance, Bradley et al., (2013) demonstrated that players in the $3^{\text {rd }}$ highest league in English soccer covered more high-intensity running distance than those in the $1^{\text {st }}$ and $2^{\text {nd }}$ highest standard but performed less passes and successful passes. Despite these identified performance differences, the physical capacity of players in these leagues was shown to be similar. Other researchers have suggested that technical rather than physical indicators differentiate between various league rankings and/or competitive standards in elite soccer (Carling, 2013; Castellano et al., 2011). Whilst these groups propose that physical indicators are not associated with league ranking, they do emphasise that they could enable the maintenance of technical proficiency (Carling \& Dupont, 2011; Rampinini et al., 2008), and thus should not be ignored as contributors to overall performance. Despite the complex inter-play between physical and technical indicators in elite soccer, no research currently 
exists that uses an integrated approach to analyse how both have changed across a longitudinal period in relation to league ranking.

The English Premier League (EPL) is arguably one of the most competitive Leagues in the world and over the last decade has undergone substantial change with the distances covered at high-intensity and sprinting increasing by $30-50 \%$ and the number of passes rising by $40 \%$ (Barnes, Archer, Hogg, Bush, \& Bradley, 2014). Similarly, research has found position-specific evolutionary match performance trends in the EPL (Bush, Barnes, Archer, Hogg, \& Bradley, 2015), although it seems this series of studies did not account for the influence of playing standard on longitudinal patterns and thus more work in this area is needed. Studies quantifying the evolution of the game usually report the absolute and relative differences in physical and technical indicators across a large number of seasons (Wallace \& Norton, 2014; Williams, Lee, \& Reilly, 1999). Although this methodological approach may provide some insight into the evolution of game demands (Norton, Craig, \& Olds, 1999), it does have limitations. No optimal method exists based on the research literature, but a potential way to gain an understanding of evolving patterns of play is to not only track data trends across a longitudinal period but also quantify progression/regression of selected performance indicators, whilst accounting for final league ranking. Understanding how patterns of game play have evolved for sub-groups within the league may be useful to inform modifications in physical, technical and tactical preparation of players (Barnes et al., 2014; Norton et al., 1999). Moreover, as the EPL generates revenue in excess of $£ 2-3$ billion per season (Deloitte Annual Review of Football Finance, 2013), lower ranked teams will ultimately miss out on sizable financial revenue that could impact on player recruitment and infrastructural development (Oberstone, 2009). Conversely, the rewards of finishing in the top rankings include eligibility to compete in European competition such as the UEFA Champions League (UCL) and Europa League (EL) which can bring both financial and 
sporting success to clubs enabling further development. Given these identified performance and financial implications, research should determine whether a differential evolution in performance has occurred for sub-tiers within top European Domestic leagues such as the EPL. Thus, this study investigated the evolution of physical and technical performances in the EPL with special reference to final league ranking.

\section{Methods}

\section{Match Analysis and Player Data}

Match performance data were collected from seven consecutive EPL seasons (2006-07 to 2012-13) using a computerised multiple-camera tracking system (Prozone Sports Ltd $^{\circledR}$, Leeds, UK). Players' movements were captured during matches by cameras positioned at roof level and analysed using proprietary software to produce a profile of each player's physical and technical performance. The validity and reliability of this tracking system has been quantified to verify the capture process and data accuracy (Bradley et al., 2007, 2009; Di Salvo et al., 2006, 2007). For instance, the inter-observer coefficient of variation for total distance covered and high-intensity running were $<2 \%$, with the exception of sprinting, for which it was 3.5\% (Bradley et al., 2009). Furthermore, Bradley et al. (2007) observed excellent inter- and intra-observer agreement for the number and type of recorded technical events $(k>0.9)$. Ethical approval was obtained from the appropriate institutional committee with Prozone Sports $\mathrm{Ltd}^{\circledR}$ supplying the data and granting permission to publish.

Data were derived from Prozone's Trend Software and consisted of 1036 individual players across 22846 player observations. Original data files were de-sensitized and included 33 different teams overall with all 20 teams evaluated in each season. Individual match data were only included for outfield players that had completed the entire $90 \mathrm{~min}$ and matches were excluded if a player dismissal occurred (Carling \& Bloomfield, 2010). The numbers of 
observations were substantially different across season (2006-07 to 2012-13), phase of season (Aug-Nov, Dec-Feb, Mar-May), position (Attackers, Central Defenders, Central Midfielders, Full Backs, Wide Midfielders) and location (Home and Away). The original data were resampled using a stratification algorithm in order to balance the observations for all factors above, thus minimising errors when applying statistical tests. The re-sampling was achieved using the stratified function in the $\mathrm{R}$ package devtools ( $\mathrm{R}$ Development Core Team) according to the procedures of Wickham \& Chang (2013) with 14700 player observations included for further analysis. The reader is referred to Barnes et al. (2014) for a detailed breakdown of the sample.

\section{League Ranking Classifications}

Final league rankings were classified into Tiers: (A) $1^{\text {st }}-4^{\text {th }}$ ranking $(n=2519)$, (B) $5^{\text {th }}-8^{\text {th }}$ ranking $(n=2965),(C) 9^{\text {th }}-14^{\text {th }}$ ranking $(n=4448)$ and (D) $15^{\text {th }}-20^{\text {th }}$ ranking $(n=4768)$. League ranking classification is a complex process due to intra- and inter-season performance variations but a generic system was used to enable the evolution of league ranking to be explored. Tier A included the top four teams in each season that can potentially qualify for the UCL (although not guaranteed automatic qualification), while Tier B encompassed the next four teams that are on the periphery of European qualification through either the UCL or the EL. For Tier A, the top 4 teams in the EPL automatically qualified for the UCL each season except for the 2011-12 season were only the top three teams qualified. This was due to a Tier B team (finishing $6^{\text {th }}$ ) that season winning the UCL (automatically qualifying as defending champions). Tier D consisted of the bottom six teams that are typically battling against relegation. Tier $\mathrm{C}$ made up the remaining six teams that were not challenging for European qualification or battling relegation. The point's differential between the various Tiers was also calculated in each of the seven EPL seasons. 


\section{Match Performance Characteristics}

Physical indicators were coded into the following activities: standing $\left(0-0.6 \mathrm{~km} \cdot \mathrm{h}^{-1}\right)$, walking $\left(0.7-7.1 \mathrm{~km} \cdot \mathrm{h}^{-1}\right)$, jogging $\left(7.2-14.3 \mathrm{~km} \cdot \mathrm{h}^{-1}\right)$, running $\left(14.4-19.7 \mathrm{~km} \cdot \mathrm{h}^{-1}\right)$, high-speed running $\left(19.8-25.1 \mathrm{~km} \cdot \mathrm{h}^{-1}\right)$ and sprinting $\left(>25.1 \mathrm{~km} \cdot \mathrm{h}^{-1}\right.$; Bradley et al., 2009). Total distance represented the summation of distances in all categories. High-intensity running consisted of the combined distance in high-speed running and sprinting $\left(\geq 19.8 \mathrm{~km} \cdot \mathrm{h}^{-1}\right)$ and was separated into three subsets based on the teams' possession status: with (WP) or without ball possession (WOP) and when the ball was out of play (Di Salvo, Gregson, Atkinson, Tordoff, \& Drust, 2009). An explosive sprint is where a player enters a sprint immediately after a low-moderate speed activity $\left(<19.8 \mathrm{~km}^{-1}\right)$ in the previous $0.5 \mathrm{~s}$ period, without entering a high-speed run. A leading sprint is where a player enters a sprint from a high-speed run in the previous $0.5 \mathrm{~s}$ period (Di Salvo, Baron, Gonzalez-Haro, Gormasz, Pigozzi, \& Bachl, 2010). Match analysis included the coding of technical indicators based on the criteria defined by Prozone and included the number of passes, received passes, successful passes, average touches per possession and individual possessions won/lost (Hughes et al., 2012). Pass distance referred to the overall length of the pass and was split into short $(\leq 10 \mathrm{~m})$, medium $(11-24 \mathrm{~m})$ and long $(\geq 25 \mathrm{~m})$.

\section{Statistical Analysis}

Two-way independent-measures analysis of variance (ANOVA) tests with sphericity assumed were undertaken to examine the interaction between tier and season. For every parameter presented in the present study, a significant interaction between these factors was identified $(P<0.001)$. Subsequently, one-way independent-measures analysis of variance (ANOVA) tests with sphericity assumed were used to compare individual data points 
obtained from each season. Dunnet's post hoc tests were used to verify localised differences relative to 2006-07 for each subsequent season with significance set at $P<0.05$. Normality was assessed visually, since even minor deviations from normality can result in data being classified as not normally distributed. This is especially true with very large sample sizes when using standard normality tests such as Kolmogorov-Smirnov. The effect size (ES) was calculated to determine the meaningfulness of the difference, corrected for bias using Hedges formula and presented with $90 \%$ Confidence Intervals [CI]. Calculations of absolute change per season for selected indicators were assessed based on the $90 \% \mathrm{CI}$ of the coefficient of the slope (linear regression). The ES magnitudes were classified as trivial $(<0.2)$, small $(>0.2-$ 0.6), moderate (>0.6-1.2) and large (>1.2; Batterham \& Hopkins, 2006). All analyses were conducted using statistical software (R Development Core Team) and data visualisation was carried out using the ggplot 2 package accessed via the Deducer Interface for the R statistical programming language.

\section{Results}

\section{Physical Indicators}

Between the 2006-07 and 2012-13 seasons, moderate increases in high-intensity running distance were observed for all Tiers (Figure 1A, $P<0.001$; ES 0.81 [CI 0.76-0.87]), equivalent to 36 (CI 34-38) $\mathrm{m} \cdot$ year $^{-1}$ for each player. Increases in high-intensity running WP were moderate for Tier B (Figure 1B, $P<0.001$; ES 0.68 [CI 0.57-0.79]) and small for Tiers A, C and D $(P<0.005$; ES 0.26 [CI 0.13-0.38], 0.41 [CI 0.31-0.51] and 0.33 [CI 0.24-0.41], respectively). These increases were equivalent to 10 (CI 5-15) $\mathrm{m} \cdot$ year $^{-1}, 24$ (CI 20-28) $\mathrm{m} \cdot$ year $^{-1}, 13(\mathrm{CI} 10-16) \mathrm{m} \cdot$ year $^{-1}$ and $9(\mathrm{CI} 7-12) \mathrm{m} \cdot$ year $^{-1}$, for Tiers A-D, respectively. Tiers A, B, C and D illustrated moderate increases in high-intensity running WOP across the seven seasons $(P<0.001$; ES: 1.00 [CI 0.86-1.13], 0.67 [CI 0.56-0.78], 0.84 [CI 0.74-0.94], 0.67 [CI 
0.58-0.76], respectively). Large increases in sprint distance were observed from the 2006-07 to 2012-13 season for Tier B (Figure 1C, $P<0.001$; ES: 1.21 [CI 1.09-1.33]), with moderate corresponding increases for Tiers A, C and D $(P<0.001$; ES: 0.74 [CI 0.61-0.87], 0.95 [CI 0.84-1.05] and 0.84 [CI 0.75-0.93], respectively). These increases were equivalent to 17 (CI 15-19) $\mathrm{m} \cdot$ year $^{-1}, 21$ (CI 19-23) $\mathrm{m} \cdot$ year $^{-1}, 16$ (CI 15-18) m $\cdot$ year $^{-1}$ and 14 (CI 12-15) m $\cdot$ year $^{-1}$, for Tiers A-D, respectively. A large increase in the total number of sprinting actions was found for all Tiers from the 2006-07 to 2012-13 seasons $(P<0.001$; ES: 1.20 [CI 1.07-1.34], 1.74 [CI 1.61-1.87], 1.41 [CI 1.30-1.52], 1.44 [CI 1.35-1.54] for A-D, respectively). These increases were equivalent to 3.7 (CI 3.4-4.0), 4.3 (CI 4.0-4.6), 3.9 (CI 3.6-4.1) and 3.3 (CI 3.1-3.5) more sprints per year, for Tiers A-D, respectively. For all Tiers, the number of leading sprints demonstrated moderate increases $(P<0.001$; ES: 0.80 [CI 0.67-0.93], 1.14 [CI 0.02-1.25], 0.91 [CI 0.81-1.02], 0.83 [CI 0.74-0.92] for A-D, respectively), equivalent to 1.4 (CI 1.3-1.4) more leading sprints per year. In comparison, large increases in the number of explosive sprints were observed ( $P<0.001$; ES 1.44 [CI 1.30-1.58], 2.01 [CI 1.88-2.14], 1.73 [CI 1.62-1.85] and 1.89 [CI 1.79-2.00], for tiers A-D, respectively), equivalent to 2.4 (CI 2.32.4) more explosive sprints being performed per year. In relative terms, the proportion of explosive sprints increased by moderate to large magnitudes over the 7-season period $(P<0.001$; ES 1.02 [CI 0.88-1.15], 1.36 [CI 1.24-1.48], 1.36 [CI 1.25-1.46] and 1.36 [CI 1.261.46], for A-D, respectively). Average distance covered per sprint decreased from 2006-07 to 2012-13 by a moderate magnitude for all Tiers $(P<0.001$; ES: 0.76 [CI $0.63-0.89], 0.88$ [CI 0.77-1.00], 0.96 [CI 0.86-1.06], 0.93 [CI 0.84-1.02], for A-D respectively), with an overall 0.16 (CI $0.15-0.16) \mathrm{m}$ decrease in the average distance covered during each sprint per year across the seven seasons. 
A large increase was observed in Tier B for the number of passes performed $(38 \pm 16$ vs. 21 $\pm 10, P<0.001$; ES: 1.34 [CI 1.22-1.46]) and received (32 \pm 14 vs. $15 \pm 8, P<0.001$; ES: 1.56 [CI 1.43-1.68]) in the 2012-13 compared to the 2006-07 season. This was equivalent to an increase of 2.8 (CI 2.6-3.0) passes made and 2.7 (CI 2.6-2.9) passes received per year in Tier B. In contrast, only small-to-moderate increases were evident for passes performed and

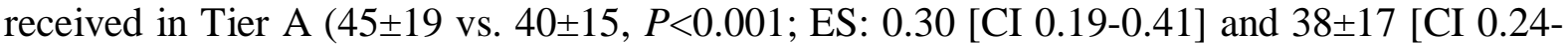
$0.44]$ vs. $33 \pm 14, P<0.001$; ES: 0.38 [CI $0.25-0.50]$ ), Tier C (33 \pm 17 vs. $27 \pm 12, P<0.001$; ES: 0.45 [CI $0.35-0.55]$ and $27 \pm 14$ vs. $20 \pm 11, P<0.001$; ES: 0.53 [CI $0.43-0.63$ ]) and Tier D (30 \pm 14 vs. $21 \pm 11, P<0.001$; ES: 0.70 [CI $0.61-0.79$ ] and $24 \pm 12$ vs. $15 \pm 9, P<0.001$; ES: 0.88 [CI 0.79-0.97]), respectively. These increases were equivalent to 0.9 (CI 0.6-1.2), 1.2 (CI 1.01.4) and 1.5 (CI 1.3-1.6) additional passes per year being made and to 1.1 (CI 0.8-1.3), 1.2 (CI 1.1-1.4) and 1.5 (CI 1.4-1.7) additional passes being received per year for Tiers A, C and $\mathrm{D}$, respectively. A moderate increase in the percentage of successful passes was observed in 2012-13 compared to $2006-07$ for Tier B (83.9 \pm 8.6 vs. $74.6 \pm 12.9 \%$; $P<0.001$; ES: 0.84 [CI $0.72-0.95])$ and Tier D (81.4 \pm 11.3 vs. $73.2 \pm 13.4 \% ; P<0.001 ;$ ES: 0.66 [CI $0.57-0.75])$, equivalent to 1.5 (CI 1.3-1.6) and 1.4 (CI 1.3-1.6) percent improvement per year. Small increases in pass success rates were observed for Tier A (87.3 \pm 7.7 vs. $84.3 \pm 8.5 \% ; P<0.001$; ES: 0.38 [CI $0.25-0.50])$ and Tier C (82.2 \pm 10.3 vs. $78.1 \pm 11.3 \%$; $P<0.001$; ES: 0.38 [CI 0.280.48]; Figure 2), equivalent to 0.7 (CI 0.5-0.8) and 0.7 (CI 0.6-0.9) annual percentage increase, respectively. The percentage of players with a pass success rate of less than $70 \%$ reduced from 7.2 to $3.1 \%$ (Tier A), 30.5 to $6.2 \%$ (Tier B), $21.0 \%$ to $11.5 \%$ (Tier C) and 34.7 to $13.4 \%$ (Tier D) between 2006-7 to 2012-13 (Figure 2).

Whilst the number of short (Table 1, $P<0.001$; ES: 1.06 [CI 0.94-1.17]) and medium passes $(P<0.001$; ES: 1.32 [CI 1.20-1.44]) in Tier B followed a similar pattern to total passes with moderate-to-large increases, the number of long passes increased by only a small 
magnitude from $6 \pm 4$ vs. $7 \pm 5(P<0.001$; ES 0.30 [CI 0.19-0.41]). The number of short, medium and long passes increased annually by 0.8 (CI 0.7-0.9), 1.8 (CI 1.7-2.0), 0.2 (CI 0.20.3) for Tier B, respectively. However, less pronounced changes were evident for Tiers A, C and D for the number of short $(P<0.001$; ES: 0.48 [CI 0.35-0.61], 0.34 [CI 0.24-0.44], 0.65 [CI 0.56-0.74]) and medium passes $(P<0.05$; ES: 0.21 [CI 0.09-0.34], 0.46 [CI 0.36-0.56], 0.70 [CI 0.61-0.79]). This was equivalent to an annual increase of 0.5 (CI 0.4-0.6), 0.3 (CI 0.2-0.3), 0.5 (CI 0.4-0.5) for short passes and 0.5 (CI 0.3-0.7), 0.8 (CI 0.7-0.9), 0.9 (CI 0.81.0) for medium passes in Tiers A, C and D, respectively. Additionally, there were minimal changes in the number of long passes across time for Tiers A, C and D.

\section{Points Differential Between League Ranking Classifications}

The demarcation line between $4^{\text {th }}$ (bottom of Tier A) and $5^{\text {th }}$ ranking (top of Tier B) in the 2006-07 season was 8 points but this decreased to just 1 point in the 2012-13 season. In contrast, the difference in points between both $8^{\text {th }}$ (bottom of Tier B) and 9th ranking (top of Tier C) and $14^{\text {th }}$ (bottom of Tier C) and $15^{\text {th }}$ ranking (top of Tier D) was $<3$ points across the same seasons (Figure 3A). The average number of points accumulated by all teams in Tiers A and B in the 2006-07, 2007-08 and 2008-09 seasons indicated that the differential between Tiers A and B was 23 points on average but in the 2010-11, 2011-12, 2012-13 seasons it decreased to 18.3 points on average. In contrast, the average points differentials between Tiers B and C in the 2006-07, 2007-08 and 2008-09 seasons (11.7 points) were similar to the average of the 2010-11, 2011-12, 2012-13 seasons (13 points). For Tiers C and D, the points differential generally decreased from an average of 12.3 points in 2006-07, 2007-08 and 2008-09 seasons to 9.3 points on average in the 2010-11, 2011-12, 2012-13 seasons. Based on linear regression analysis for changes over time within each Tier, teams finishing in Tier A accrued on average 0.43 fewer points season-on-season, with those finishing in Tier B 
amassing 0.32 points more per season. Similarly, Tier C clubs achieved 0.31 fewer points per season whilst those in Tier D accrued 0.20 points more (Figure 3B). Overall, these data would tend to indicate that the points differential between Tiers A and B and between Tiers C and $\mathrm{D}$ decreased over the seven seasons in question.

\section{Discussion}

The present study is the first to map the evolution of physical and technical parameters related to final EPL ranking and builds on the findings from recent longitudinal studies (Barnes et. al., 2014; Bush et al., 2015). It was envisaged that the present study would improve our understanding of evolving patterns of play according to final league ranking and which potentially differentiate contemporary performance.

For all Tiers, the most pronounced increases in physical performance were for the explosive metrics such as high-intensity running and sprinting. Between the 2006-07 and 2012-13 seasons, the greatest relative increase in high-intensity running distance was observed for Tier B (37\%), followed by Tiers A, C and D (33, 32 and 23\%, respectively). Similar trends were also observed for explosive metrics when year-on-year changes were calculated discounting that a one off-season had caused the patterns observed between Tiers A-D. This finding is particularly relevant as the distance covered at high-intensity is a useful measure of physical performance during match play given its association with physical capacity and its ability to demarcate between position and gender (Bradley et al., 2011, 2014; Krustrup et al., 2003, 2005). Furthermore, the reported increase for high-intensity running distance across all Tiers lies outside the reported inherent match-to-match variability for this variable in the EPL (Bush, Archer, Hogg \& Bradley, 2015; Gregson, Drust, Atkinson, \& Di Salvo, 2010). Thus, it is reasonable to conclude that the physical demands of the EPL have evolved for all Tiers, albeit at different rates. 
The data demonstrates that all Tiers covered more high-intensity running distance in possession of the ball during the 2012-13 compared to 2006-07 season. However, noticeable inter-tier differences are evident, with Tier A increasing by just $15 \%$ whereas Tier B increased by $50 \%$. Indeed, whereas at the start of the study, Tier A teams covered markedly greater high-intensity running distances in possession of the ball than Tier B, by 2012-13 distances covered were virtually identical. This observation is perhaps mirrored by the fact that, over the period of the study, distances covered out of possession of the ball by Tier A clubs increased by $43 \%$ with Tier B, C and D clubs increasing by 26,35 and $26 \%$, respectively. This could be reflective of a reduction in tactical and territorial dominance of Tier A clubs relative to those finishing in Tier B or simply that the top clubs are unafraid of leaving possession to lower-ranked peers as they feel they have the physical, tactical and/or technical ability to cope. It could however, also be indicative of Tier A clubs being more technically and tactically efficient in possession of the ball than their lower Tier counterparts, with a resultant reduction in non-productive high-intensity efforts. The reader should be aware of the descriptive nature of the current study and that the above reasons have yet to be verified within the literature and thus future research should continue to examine the impact these technical and tactical factors have on physical metrics. This trend seems to be an evolutionary shift in the high-intensity running patterns in Tiers $\mathrm{A}$ and $\mathrm{B}$, but the general trend that higher ranked teams cover more high-intensity running while in possession of the ball compared to lower ranked teams (Di Salvo et al., 2009; Rampinini et al., 2009) still holds true when observing trends across Tiers A-B versus C-D in the 2012-13 season.

The number of sprints and the distance covered sprinting increased dramatically between 2006-07 and 2012-13. The patterns across Tiers mirrored those found for highintensity running, with the most marked increase in sprinting distance (70\%) and number of sprints (107\%) found within Tier B. The relatively greater increase in the number of sprints to 
distance covered sprinting for all Tiers is reflective of the fact that average distance per sprint decreased between 2006-07 (6.8-7.0 $\mathrm{m})$ and 2012-13 (5.8-6.0 $\mathrm{m})$. This has implications for developing training that not only mimics these short intense bouts but also conditioning to cope with rapid accelerations and decelerations to reduce the propensity of injury (Petersen, Thorborg, Nielsen, Budtz-Jørgensen, \& Hölmich, 2011).

It has previously been reported that the absolute number of explosive and leading sprints in match play is position-specific (Di Salvo et al., 2009, 2010) and that a positionspecific evolution in this sprint profile has occurred (Bush et al., 2015). The present findings demonstrate that the increase in the absolute numbers of leading and explosive sprints is also Tier-specific. Tier B demonstrated the greatest increase in both leading (68\%) and explosive (180\%) sprints, indicating that the physical performances of clubs in this Tier have evolved to a greater extent than their counterparts. Attributing these findings to any single factor is difficult but they could be related to the style of play/tactical system utilised by Tier B teams as these impact physical performances (Bradley et al., 2011; Bush et al., 2015) or possibly the recruitment of players with more explosive characteristics. Given the sub-maximal nature of soccer, whereby players work well within their physical capacity (Paul, Bradley \& Nassis, 2015), the increase in physical performance of Tier B clubs could be related to added incentives to push for a top four position given the financial rewards now afforded to Tier A (Deloitte Annual Review of Football Finance, 2013; Oberstone, 2009). Finally, previous research has proposed an association between volume of sprinting in match play and hamstring injury risk (Small, McNaughton, Greig, Lohkamp, \& Lovell, 2009). Although no evidence exists of injury occurrence by Tier, the present data would suggest those playing for clubs finishing in Tier B might have been at greater risk of injury and may therefore benefit more from appropriate injury prevention strategies but all Tiers should utilise such an approach. Interestingly, although differences between Tiers for increases in absolute numbers 
of sprints were identified, the relative proportion of explosive sprints was very similar in 2006-07 ( 34-36\%) and in 2012-13 ( 44-46\%), suggesting homogeneous development across the league.

The addition of technical performance parameters provides a further layer of granularity to this analysis and falls in line with other studies (Barnes et al., 2014; Bush et al., 2015). Over the period of this study, Tier A consistently demonstrated the greatest number of technical events and the highest levels of technical performance (e.g. number of passes and successful passes), a rather unsurprising fact given that we would normally expect the most technically competent teams to finish highest in the league. In season 2006-07, Tier A recorded almost double the number of passes made (40) and passes received (32) than Tiers B (21 and 15, respectively), C (27 and 20, respectively) and D (21 and 15, respectively). However, over the seven season period of this study the greatest increases in these parameters ( $81 \%$ and $113 \%$ for passes made and passes received, respectively) was demonstrated by Tier $\mathrm{B}$, resulting in the technical performance gap between Tier A and Tier B being significantly reduced. This is further supported by improvements in Tier B's pass success rate (12\%) and numbers of short (50\%) and medium (50\%) passes. These developments are greater than for Tier A (4, 33 and 13\%, respectively), Tier C (5, 13 and 36\%, respectively) and Tier D (11, 38 and $45 \%$, respectively). In addition, the percentage of player occurrences with a passing success rate of $<70 \%$, identified as a minimum requirement in elite soccer (Dellal et al., 2011) decreased dramatically over the seven seasons, most notably in Tier B where it reduced from 31 to $6 \%$. The convergence of Tiers $\mathrm{A}$ and $\mathrm{B}$ in relation to technical performance is somewhat mirrored with the lower Tiers, with the improvements in technical performance for Tier D being greater than that for Tier C.

A global measure of performance evolution of the different sub-components within the EPL is the average points scored by teams finishing in individual Tiers. It is interesting to 
note that these trends very closely mirror those observed for the physical and technical metrics, with a convergence of points accumulated in Tiers A and B, and of Tiers C and D. Indeed, teams finishing in Tier A have accrued on average 0.4 points less season-on-season, with those finishing in Tier B amassing 0.3 points more. Similarly, Tier C clubs won 0.3 points per season less whilst those in Tier D accrued 0.2 points more. Whilst it would be naïve to assume that these relationships are causative, the association is nevertheless of great interest and perhaps worthy of further investigation. It is also important to note that if Tier B

is closing the gap on Tier A, this may also mean a widened gap between Tiers B and C which may have implications for clubs with aspirations of breaking into a higher Tier. In summary, the net result of these observations reveals a league which is evolving both physically and technically, but one in which the greatest pace of change has occurred in teams finishing between $5^{\text {th }}$ and $8^{\text {th }}$ place.

\section{Acknowledgements}

Thanks to Prozone Sports for providing access to the data used in this study. 


\section{References}

Andersson, H., Ekblom, B., \& Krustrup, P. (2008). Elite football on artificial turf versus natural grass: movement patterns, technical standards, and player impressions. Journal of Sports Sciences, 26(2), 113-22.

Apor, P. (1988). Successful Formulae for Fitness Testing. In T. Reilly, A. Lees, K. Davids, \& W. Murphy (Eds.), Science and Football (pp. 95-107). Abingdon, UK: Routledge.

Barnes, C., Archer, D., Hogg, B., Bush, M., \& Bradley, P. S. (2014). The Evolution of Physical and Technical Performance Parameters in the English Premier League. International Journal of Sports Medicine, 35(13), 1095-1100.

Batterham, A. M., \& Hopkins, W. G. (2006). Making meaningful inferences about magnitudes. International Journal of Sports Physiology and Performance, 1(1), 50-57.

Bradley, P. S., Carling, C., Archer, D., Roberts, J., Dodds, A., Di Mascio, M., ... Krustrup, P. (2011). The effect of playing formation on high-intensity running and technical profiles in English FA Premier League soccer matches. Journal of Sports Sciences, 29(8), 821-830.

Bradley, P. S., Carling, C., Diaz, A. G., Hood, P., Barnes, C., Ade, J., ... Mohr, M. (2013). Match performance and physical capacity of players in the top three competitive standards of English professional soccer. Human Movement Science, 32(4), 808-821.

Bradley, P. S., Dellal, A., Mohr, M., Castellano, J., \& Wilkie, A. (2014). Gender differences in match performance characteristics of soccer players competing in the UEFA Champions League. Human Movement Science, 33(1), 159-71. 
Bradley, P. S., \& Noakes, T. D. (2013). Match running performance fluctuations in elite soccer: indicative of fatigue, pacing or situational influences? Journal of Sports Sciences, 31(15), 1627-38.

Bradley, P. S., O’Donoghue, P., Wooster, B., \& Tordoff, P. (2007). The reliability of Prozone MatchViewer: a video-based technical performance analysis system. International Journal of Performance Analysis in Sport, 7 (3), 117-129.

Bradley, P. S., Sheldon, W., Wooster, B., Olsen, P., Boanas, P., \& Krustrup, P. (2009). Highintensity running in English FA Premier League soccer matches. Journal of Sports Sciences, 27(2), 159-168.

Bush, M., Barnes, C., Archer, D., Hogg, B., \& Bradley, P. S. (2015). Evolution of Match Performance Parameters for Various Playing Positions in the English Premier League. Human Movement Science, 39(1), 1-11.

Bush, M., Archer, D., Hogg, B., \& Bradley, P. S. (2015). Factors Influencing Physical and Technical Variability in the English Premier League. International Journal of Sports Physiology and Performance, (Epub ahead of print).

Carling, C. (2013). Interpreting Physical Performance in Professional Soccer Match-Play: Should We be More Pragmatic in Our Approach? Sports Medicine, 43(8), 655-663.

Carling, C., \& Bloomfield, J. (2010). The effect of an early dismissal on player work-rate in a professional soccer match. Journal of Science and Medicine in Sport, 13(1), 126-128.

Carling, C., \& Dupont, G. (2011). Are declines in physical performance associated with a reduction in skill-related performance during professional soccer match-play? Journal of Sports Sciences, 29(1), 63-71. 
Castellano, J., Blanco-Villasenor, A., \& Alvarez, D.. (2011). Contextual variables and timemotion analysis in soccer. International Journal of Sports Medicine, 32(6), 415-421.

Dellal, A., Chamari, K., Wong, D.P., Ahmaidi, S., Keller, D., Barros, R., Bisciotti G.N., \& Carling C. (2011). Comparison of physical and technical performance in European soccer match-play: FA Premier League and La Liga. European Journal of Sport Science, 11(1): 5159.

Deloitte. (2013). Annual Review of Football Finance. Retrieved from www.deloitte.co.uk/sportsbusinessgroup.

Di Salvo, V., Baron, R., Tschan, H., Calderon Montero, F. J., Bachl, N., \& Pigozzi, F. (2007). Performance characteristics according to playing position in elite soccer. International Journal of Sports Medicine, 28(3), 222-227.

Di Salvo, V., Collins, A., McNeill, B., \& Cardinale, M. (2006). Validation of Prozone: A new video-based performance analysis system. International Journal of Performance Analysis in Sport, 6(1), 108-119.

Di Salvo, V., Gregson, W., Atkinson, G., Tordoff, P., \& Drust, B. (2009). Analysis of High Intensity Activity in Premier League Soccer. International Journal of Sports Medicine, 30(3), $205-212$.

Di Salvo, V., Baron, R., Gonzalez-Haro, C., Gormasz, C., Pigozzi, F., \& Bachl, N. (2010). Sprinting Analysis of Elite Soccer Players During European Champions League and UEFA Cup Matches/ Journal of Sports Sciences, 28(14), 1489-1492. 
Gregson, W., Drust, B., Atkinson, G., \& Di Salvo, V. (2010). Match-to-match variability of high-speed activities in premier league soccer. International Journal of Sports Medicine, $31(4), 237-242$.

Hughes, M., Caudrelier, T., James, N., Donnelly, I., Kirkbride, A., \& Duschesne, C. (2012). Moneyball and soccer - an analysis of the key performance indicators of elite male soccer players by position. Journal of Human Sport and Exercise, 7(2), 402-412.

Krustrup, P., Mohr, M., Amstrup, T., Rysgaard, T., Johansen, J., Steensberg, A., ... Bangsbo, J. (2003). The yo-yo intermittent recovery test:physiological response, reliability and validity. Medicine and Science in Sports and Exercise, 35(4), 697-705.

Krustrup, P., Mohr, M., Ellingsgaard, H., \& Bangsbo, J. (2005). Physical demands during an elite female soccer game: importance of training status. Medicine and Science in Sports and Exercise, 37(7), 1242-1248.

Mohr, M., Krustrup, P., \& Bangsbo, J. (2003). Match performance of high-standard soccer players with special reference to development of fatigue. Journal of Sports Sciences, 21(7), $519-528$.

Mohr, M., Nybo, L., Grantham, J., \& Racinais, S. (2012). Physiological responses and physical performance during football in the heat. PloS One, 7(6), e39202.

Norton, K. I., Craig, N. P., \& Olds, T. S. (1999). The evolution of Australian football. Journal of Science and Medicine in Sport, 2(4), 389-404.

Oberstone, J. (2009). Differentiating the Top English Premier League Football Clubs from the Rest of the Pack: Identifying the Keys to Success. Journal of Quantitative Analysis in Sports, 5(3), 1-27. 
Paul, D.J., Bradley, P.S. \& Nassis, G.P. (2015). Factors affecting match running performance of elite soccer players: shedding some light on the complexity. International Journal of Sports Physiology and Performance, 10(4), 516-519.

Petersen, J., Thorborg, K., Nielsen, M. B., Budtz-Jørgensen, E., \& Hölmich, P. (2011). Preventive effect of eccentric training on acute hamstring injuries in men's soccer: a clusterrandomized controlled trial. The American Journal of Sports Medicine, 39(11), 2296-303.

Rampinini, E., Coutts, A J., Castagna, C., Sassi, R., \& Impellizzeri, F. M. (2007). Variation in top level soccer match performance. International Journal of Sports Medicine, 28(12), $1018-1024$.

Rampinini, E., Impellizzeri, F., Castagna, C., Coutts, A., \& Wisløff, U. (2009). Technical performance during soccer matches of the Italian Serie A league: Effect of fatigue and competitive level. Journal of Science and Medicine in Sport, 12(1), 227-233.

Rampinini, E., Impellizzeri, F. M., Castagna, C., Azzalin, A., Ferrari Bravo, D., \& Wisløff, U. (2008). Effect of match-related fatigue on short-passing ability in young soccer players. Medicine and Science in Sports and Exercise, 40(5), 934-942.

Small, K., McNaughton, L. R., Greig, M., Lohkamp, M., \& Lovell, R. (2009). Soccer fatigue, sprinting and hamstring injury risk. International Journal of Sports Medicine, 30(8), 573-8.

Stølen, T., Chamari, K., Castagna, C., \& Wisløff, U. (2005). Physiology of Soccer. Sports Medicine, 35(6), 501-536.

Wallace, J. L., \& Norton, K. I. (2014). Evolution of World Cup soccer final games 19662010: Game structure, speed and play patterns. Journal of Science and Medicine in Sport, $17(2), 223-228$. 
Wickham, H., \& Chang, W. (2013). Tools to make developing $R$ code easier. R Package 1.2.

Williams, A., Lee, D., \& Reilly, T. (1999). A quantitative analysis of matches played in the 1991-92 and 1997-98 seasons. London: The Football Association. 


\section{Figure Legends}

Figure 1: Box and Whisker plots with median values, interquartile ranges and outliers for the distances covered in (A) High-intensity running, (B) High-intensity running with ball possession and (C) Sprinting across seven EPL seasons for various Tiers. The larger filled circles represent outliers which are values that lie more than one and half times the length of either end of the box $(1.5 \times$ interquartile range). Line denotes regression line.

Figure 2: Visualisation of data trends using a two-dimensional kernel density plot of the number of passes and pass success rate across seven EPL seasons for various Tiers. The plots show the distribution both along the $\mathrm{x}$ and $\mathrm{y}$ axis, indicating both an increasing number of passes (data plot shifting to the right on the $\mathrm{x}$ axis) and successful passes across the seasons (data plot shifting upwards on the y axis). Plots are calculated through combining numerous Gaussian curves on the $\mathrm{x}$ and $\mathrm{y}$ axis to create the contours of each plot.

Figure 3: (A) Points differential across seven EPL seasons for various Tiers. The demarcation line between Tiers: A and B ( $4^{\text {th }}$ vs $5^{\text {th }}$ ranking or bottom of Tier A vs top of Tier B), B and C ( $8^{\text {th }}$ vs $9^{\text {th }}$ ranking or bottom of Tier B vs top of Tier C) and C and D $\left(14^{\text {th }}\right.$ vs $15^{\text {th }}$ ranking or bottom of Tier C vs top of Tier D), (B) Average points scored for teams finishing in each Tier of the league for each season. Line denotes regression line and grey area highlights the $90 \%$ confidence intervals. The data demonstrate a convergence of points accumulated in Tiers. For instance, Tiers A and C amassed fewer points season-on-season while Tiers B and D accrued more points.

\section{Table Legend}

Table 1: Passing performance across seven EPL seasons for various Tiers. Data are presented as means and standard deviations. Pass distance referred to the overall length of the pass and was split into short $(\leq 10 \mathrm{~m})$, medium (11-24 m) and long $(\geq 25 \mathrm{~m})$. Denotes difference from the 2006-07 season $(\# p<0.05, \S p<0.01 ; * p<0.001)$. 
Figure 1A:

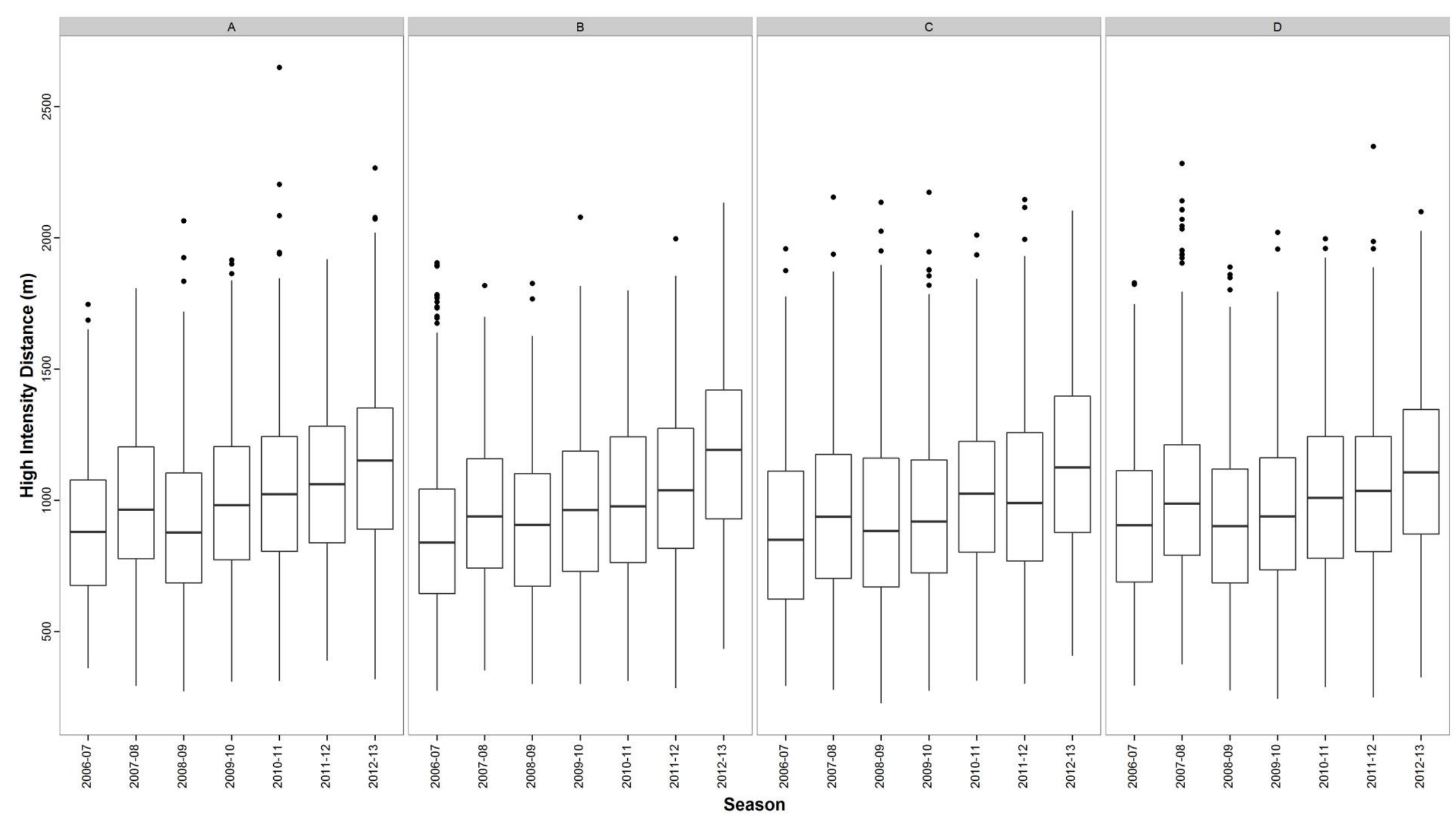


Figure 1B

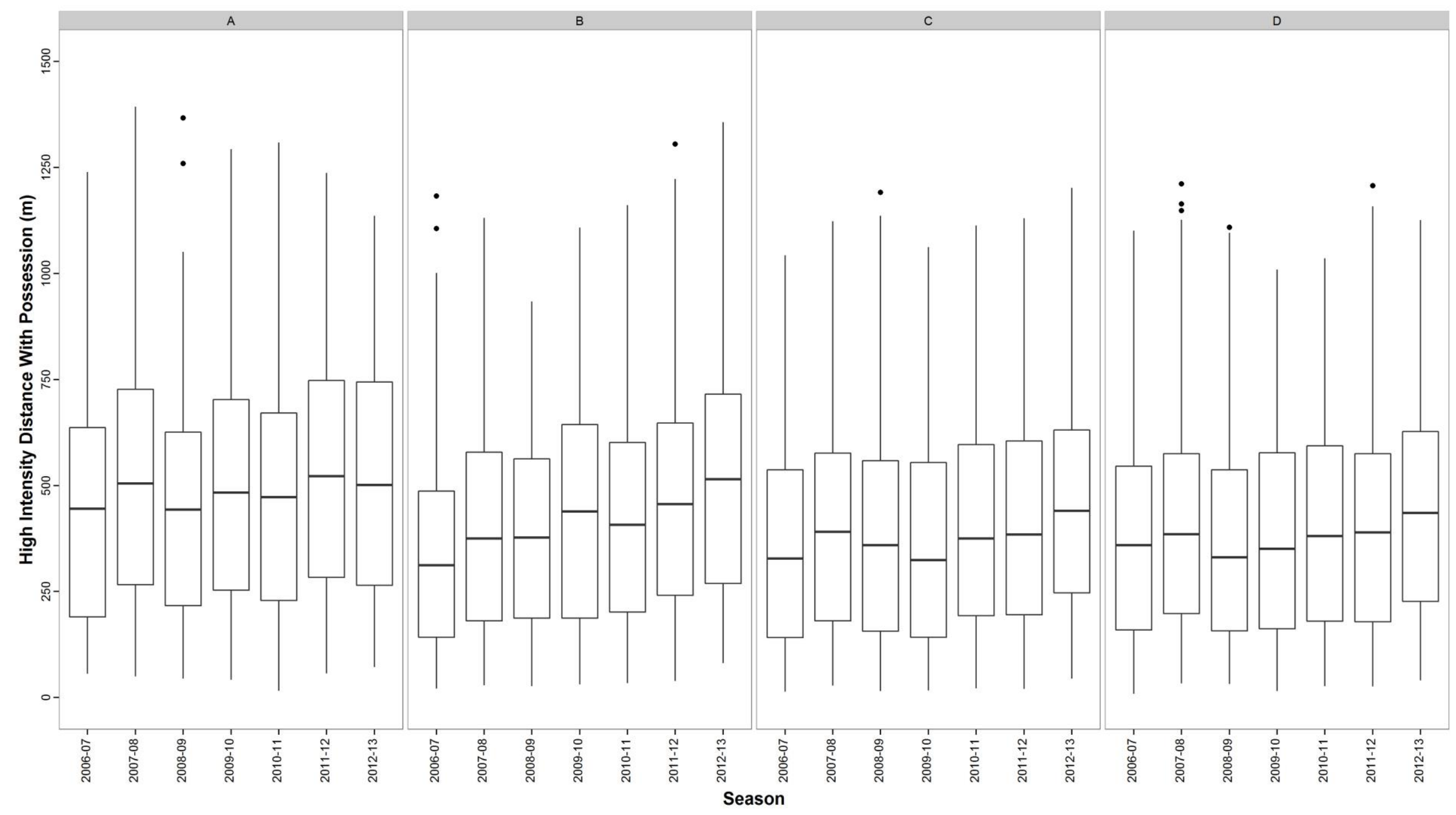


Figure 1C

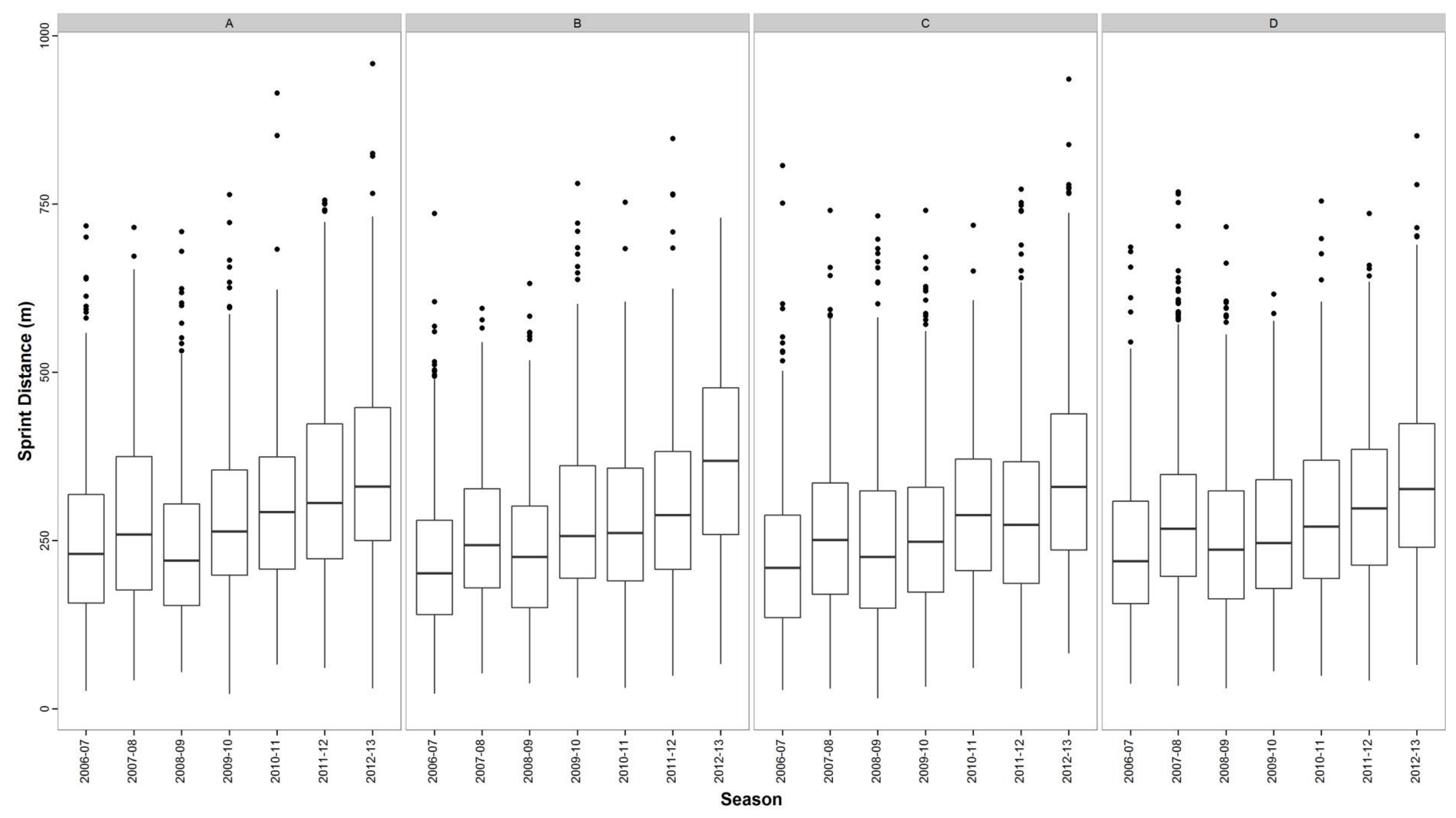




\section{Figure 2:}

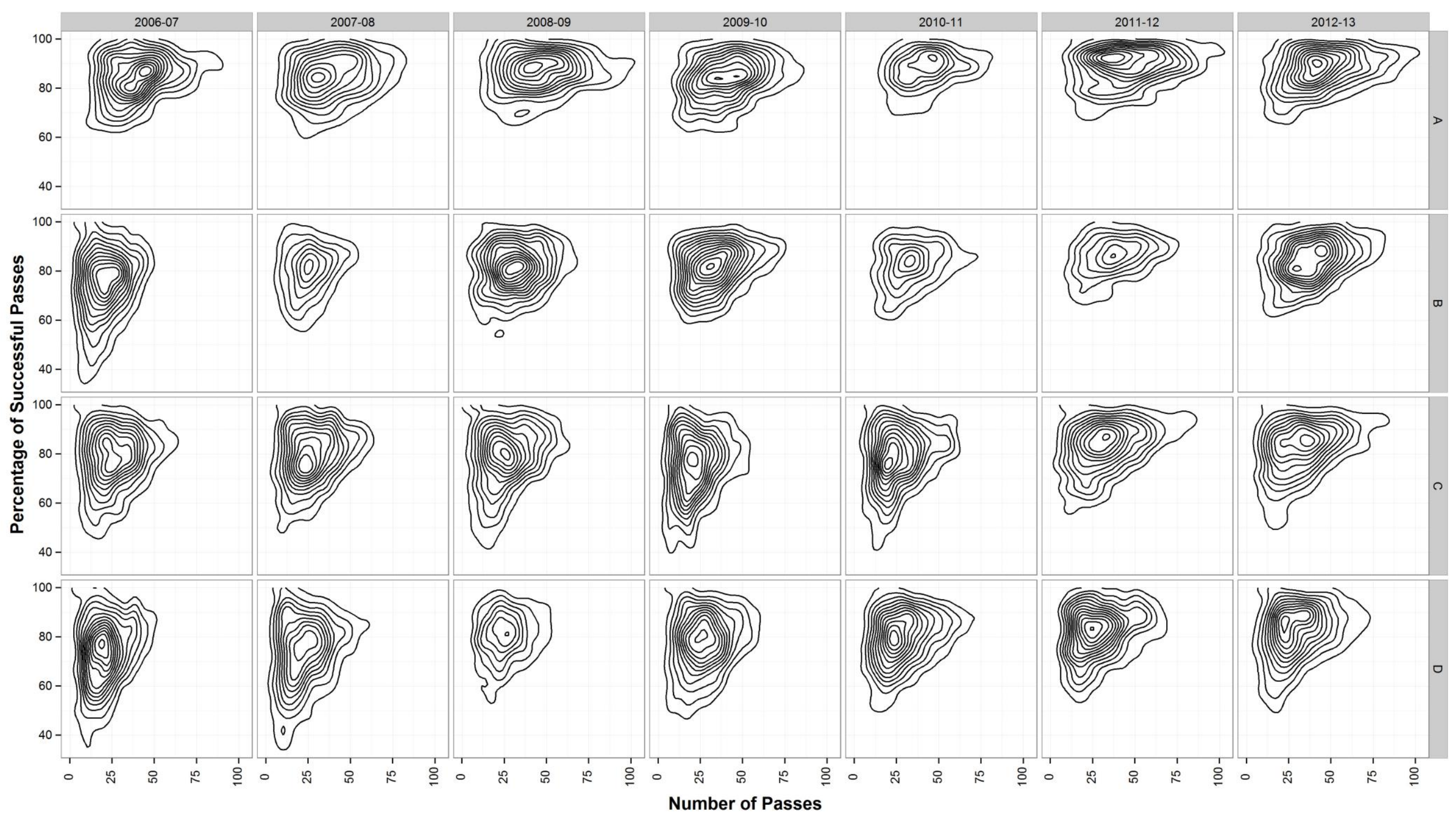


Figure 3A:

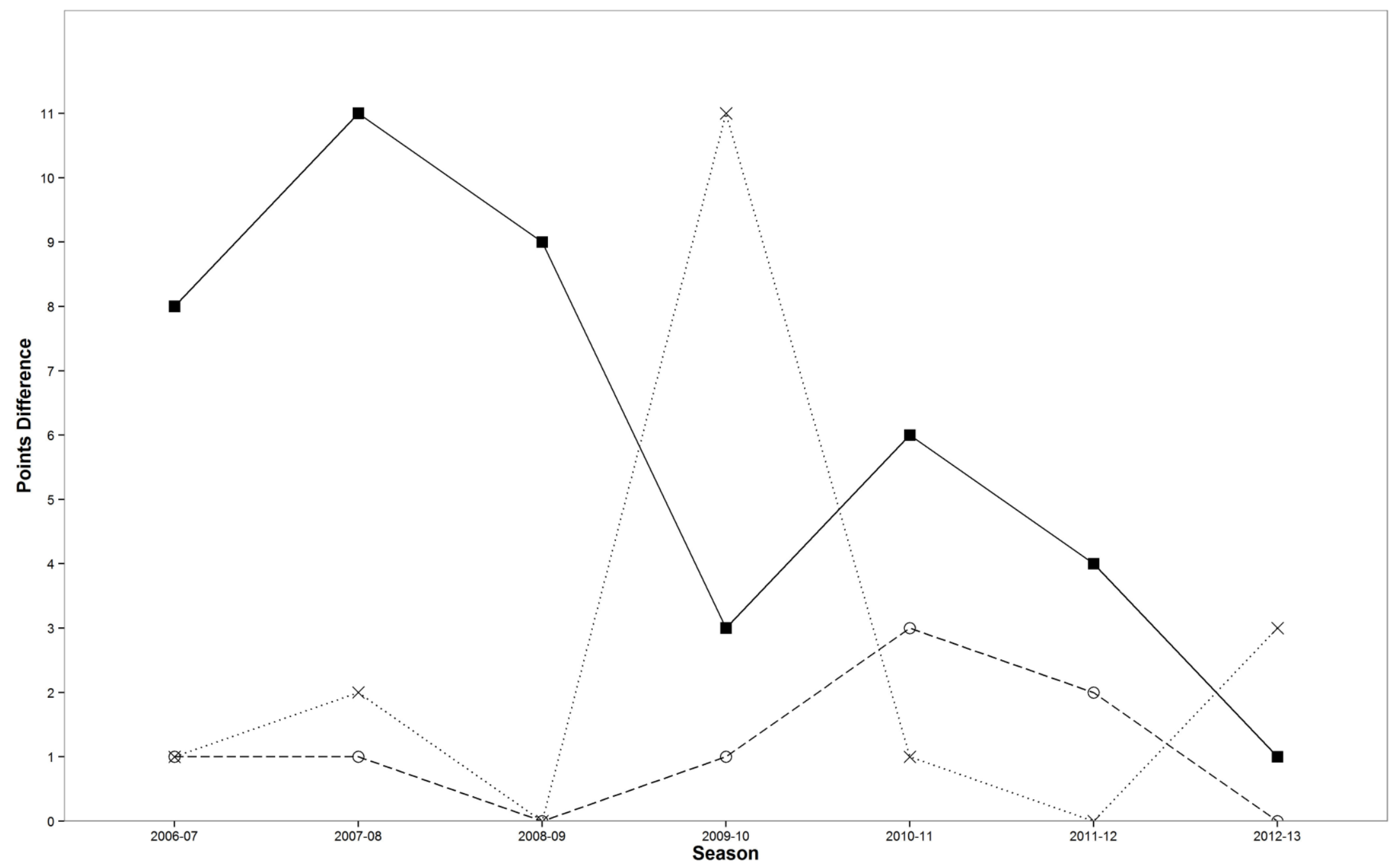

Tiers

-A - B (4th - 5th)

- $X$ B - C (8th - 9th)

$\theta$ C - D (14th - 15th) 
Figure 3B:

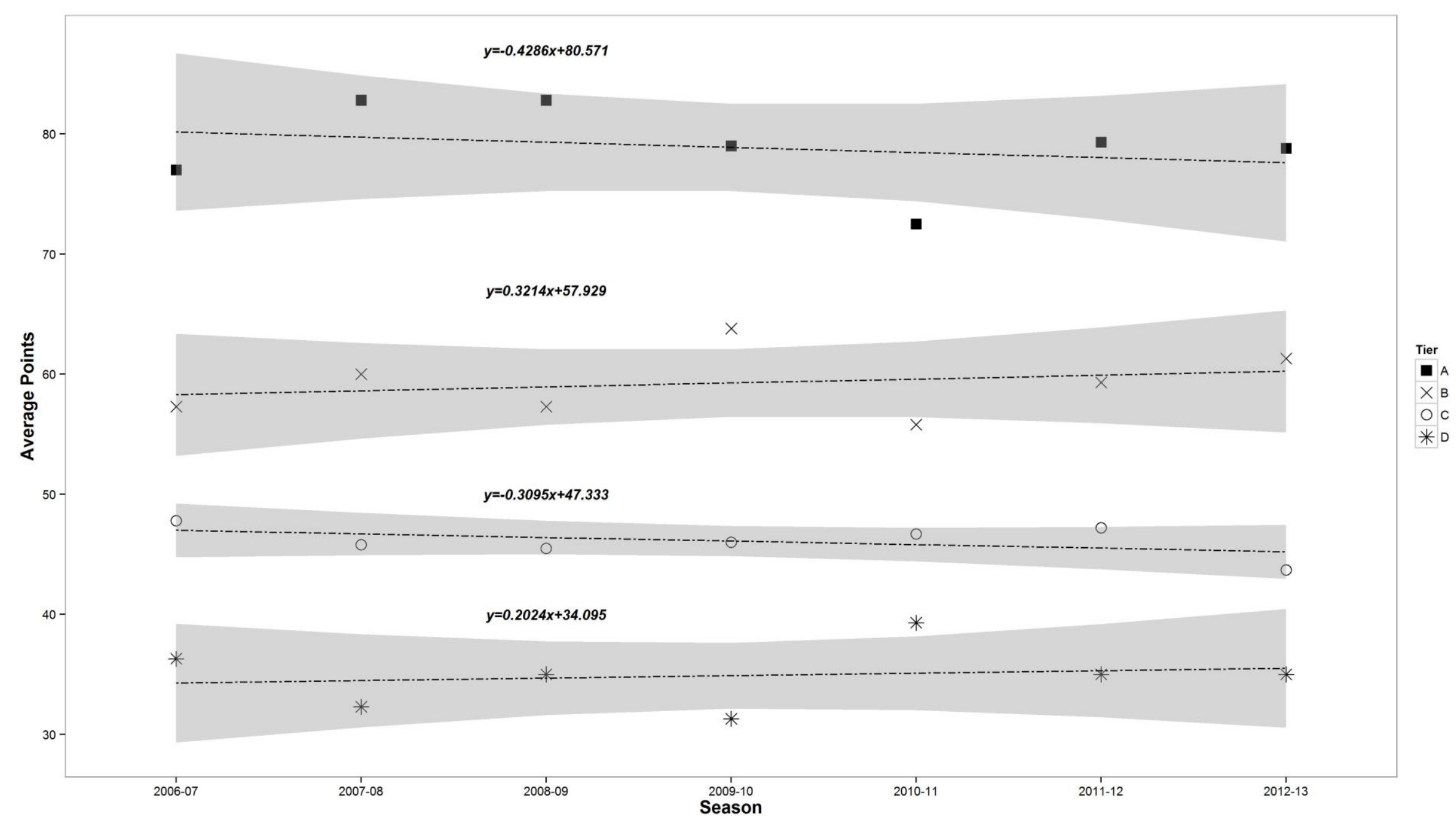


Table 1:

\begin{tabular}{|c|c|c|c|c|c|c|c|c|c|c|c|c|}
\hline & \multicolumn{4}{|c|}{ Short Passes } & \multicolumn{4}{|c|}{ Medium passes } & \multicolumn{4}{|c|}{ Long Passes } \\
\hline Season & $\mathbf{A}$ & B & $\mathrm{C}$ & D & $\mathbf{A}$ & B & $\mathrm{C}$ & D & $\mathbf{A}$ & B & $\mathbf{C}$ & D \\
\hline 2006-07 & $9.4 \pm 5.3$ & $5.0 \pm 3.5$ & $6.7 \pm 4.0$ & $5.2 \pm 3.7$ & $23.2 \pm 10.9$ & $10.6 \pm 6.1$ & $14.0 \pm 7.7$ & $10.7 \pm 6.5$ & $7.1 \pm 4.5$ & $5.5 \pm 3.7$ & $5.8 \pm 3.9$ & $5.3 \pm 3.9$ \\
\hline 2007-08 & $10.2 \pm 5.5$ & $6.7 \pm 4.4^{*}$ & $7.1 \pm 4.8$ & $5.9 \pm 4.1 \#$ & $22.7 \pm 11.4$ & $13.4 \pm 7.3^{*}$ & $14.3 \pm 7.7$ & $11.8 \pm 7.9$ & $6.3 \pm 4.6$ & $5.7 \pm 3.9$ & $5.8 \pm 3.8$ & $5.5 \pm 3.9$ \\
\hline 2008-09 & $12.0 \pm 6.5^{*}$ & $8.1 \pm 5.1 *$ & $6.9 \pm 4.9$ & $6.7 \pm 4.1^{*}$ & $26.8 \pm 12.6^{*}$ & $17.3 \pm 9.1 *$ & $14.0 \pm 8.6$ & $14.1 \pm 8.0^{*}$ & $7.5 \pm 5.9$ & $6.5 \pm 4.2^{*}$ & $5.7 \pm 4.2$ & $5.9 \pm 4.0$ \\
\hline 2009-10 & $10.6 \pm 6.1$ & $8.1 \pm 5.2^{*}$ & $6.0 \pm 4.3$ & $7.2 \pm 4.7^{*}$ & $23.0 \pm 10.5$ & $18.4 \pm 9.3^{*}$ & $11.4 \pm 7.4^{*}$ & $14.3 \pm 7.6^{*}$ & $6.4 \pm 4.4$ & $6.8 \pm 4.5^{*}$ & $5.2 \pm 3.7$ & $5.9 \pm 4.4 \#$ \\
\hline 2010-11 & $11.3 \pm 5.8^{*}$ & $8.6 \pm 5.1 *$ & $6.6 \pm 4.4$ & $7.5 \pm 4.4^{*}$ & $24.5 \pm 11.0$ & $19.2 \pm 9.7 *$ & $13.9 \pm 8.0$ & $15.5 \pm 8.7^{*}$ & $6.4 \pm 4.3$ & $6.5 \pm 4.3 \S$ & $5.7 \pm 4.1$ & $6.2 \pm 4.4^{*}$ \\
\hline 2011-12 & $13.3 \pm 7.8^{*}$ & $10.1 \pm 5.9^{*}$ & $8.5 \pm 5.6^{*}$ & $7.8 \pm 5.0^{*}$ & $26.5 \pm 13.7^{*}$ & $20.7 \pm 9.7 *$ & $18.5 \pm 11.9^{*}$ & $15.8 \pm 9.1^{*}$ & $6.7 \pm 5.0$ & $6.8 \pm 4.7^{*}$ & $6.2 \pm 4.6$ & $5.6 \pm 4.2$ \\
\hline 2012-13 & $12.4 \pm 7.1^{*}$ & $9.8 \pm 5.5^{*}$ & $8.5 \pm 5.7 *$ & $8.1 \pm 5.2^{*}$ & $25.8 \pm 13.1 \#$ & $21.8 \pm 10.6^{*}$ & $18.5 \pm 11.0^{*}$ & $16.2 \pm 9.1^{*}$ & $6.7 \pm 4.7$ & $6.8 \pm 4.9^{*}$ & $6.2 \pm 4.5$ & $5.6 \pm 4.0$ \\
\hline
\end{tabular}

Denotes significantly different from 2006-07 $(\# p<0.05, \S p<0.01, * p<0.001$ 
